\title{
Paradigma disciplinar: Las organizaciones arquitectónicas y sus plataformas de diseño y producción
}

\author{
Disciplinar paradigm: The architectural organizations and their design and production \\ platforms
}

\section{$<$ RESUMEN>}

El propósito de este ensayo es proporcionar una visión contemporánea sobre los grandes cambios que nuestra disciplina ha atravesado en el último par de décadas, en términos de organización del trabajo arquitectónico. La investigación sobre el paradigma disciplinar en este ámbito dará el marco teórico que ayudará a la definición de la estructura de la oficina de arquitectura en el plano de organización y plataformas de diseño y producción.

$<A B S T R A C T>$

The purpose of this essay is to provide a contemporary view to the great changes that our discipline has been going through in the last decades in terms of the architectural work organization. The research of the disciplinary paradigm will be the theoretical ground that is going to help to define the structure of the architectural office, in terms of purely organization and platforms for design and production.

<PALABRAS CLAVE >

DISEÑO COLABORATIVO / REDES, RIZOMA /

INTERDISCIPLINARIDAD / PLATAFORMAS DE DISEÑO

<KEY WORDS >

COLLABORATIVE DESIGN / NETWORKS, RHIZOME / INTERDISCIPLINARY / DESIGN PLATFORMS

\section{Introducción}

El trabajo arquitectónico, durante los últimos 15 ó 20 años, ha sido testigo de una serie de procesos evolutivos que se reflejan en un importante cambio en su estructura y definición. La organización de la oficina de arquitectura como la conocíamos -y como aún se manifiesta de manera general en nuestro país-, con una cabeza creativa a cargo de una serie de arquitectos que llevan a cabo las ideas del primero, en una clara organización piramidal, se ha ido transformando gradualmente en memoria del pasado. La disciplina hoy, desde una perspectiva global, se desarrolla a través de un paradigma completamente diferente, en donde los límites se encuentran cada día más difusos, y los adelantos tecnológicos agregan la sustancia necesaria para este nuevo entendimiento de la organización del trabajo arquitectónico, en términos de diseño y producción.

El presente ensayo buscará la comprensión de estas nuevas formaciones a través del concepto de rizoma desarrollado por Gilles Deleuze ${ }^{1}$, así como el entendimiento de las nuevas plataformas del trabajo arquitectónico -no sólo desde la perspectiva del rol de los software, sino que también del cambio desde el dibujo bidimensional clásico hacia el modelo digital 3D como elemento básico de la producción y el diseño arquitectónico-, indagando en el mapa organizacional actual de las disciplinas y en la idea de las comunicaciones globales, como base estructural de la producción contemporánea.

\footnotetext{
Arquitecto de la Universidad de Chile y Master in Architecture and Urbanism (Design Research Lab) Architectural Association, Londres, Reino Unido. Actualmente vive y trabaja en Londres.

Deleuze, G., Guattari, F. Thousand Plateaus: Capitalism and schizophrenia. (Traducción Brian Massumi). Londres: Deleuze, G., Guattari,
} 


\section{El paradigma disciplinar}

El paradigma de la era industrial, con su línea de producción y ensamblaje junto con el control social basado en las estructuras disciplinares, es historia. La clásica combinación de disciplinas principales con sus consecuentes secundarias en una estructura jerárquica de «árbol-raíz», es algo simplemente no funcional dada la incapacidad de este esquema para interrelacionar diferentes disciplinas. No resulta coherente ni productiva en la nueva era de la información. Es así como hoy en día la colaboración entre diversas áreas disciplinares, se convierte en uno de los motores fundamentales para el desarrollo y crecimiento de los diferentes campos del conocimiento.

En este contexto, los avances tecnológicos de la última mitad de siglo resultan ser los principales catalizadores de tales cambios. Una de las primeras personas en referirse al tema fue Marshall McLuhan ${ }^{2}$, cuando en los sesentas escribió sobre el fenómeno actual de estas nuevas organizaciones sociales colectivas basadas en la tecnología de las telecomunicaciones, llamando a este modelo como aldea global. Pero quizás el evento que hizo esta situación más tangible para toda la sociedad fue la presentación de Doug Engelbart ${ }^{3}$, en el auditorio cívico de San Francisco, en 1968. Su presentación de 30 minutos sobre una nueva interface con las máquinas (computadores) fue el primer indicio de lo que hoy podemos llamar espacio de información ${ }^{4}$. Esta interface, comúnmente conocida como la GUI (Graphic User Interface), es lo que hoy en día hace posible concebir la tecnología de la información como un legítimo espacio de interacción, más que como un simple conjunto de máquinas; un verdadero medioambiente, un paisaje, un «espacio virtual» a explorar.

Así, la aparición y desarrollo de las tecnologías de información han transformado a la transferencia de datos; la reorganización de las estructuras del conocimiento y el movimiento a través de diferentes disciplinas, en algo dado y completamente asumido en el discurso contemporáneo sobre diseño. Como Piere Lèvi ha dicho: «...estas tecnologías crecientemente desmaterializan los cerrados límites de las disciplinas, haciendo del conocimiento un 'gran mosaico' en el cual cada campo puede ser envuelto por otro ${ }^{5}$. Desde este punto de vista podemos entender el paradigma multidisciplinar en el campo de la arquitectura, como un entrelazado de múltiples y diferentes conocimientos enfocados en resolver un problema arquitectónico; pero ya no con la organización de «árbol», con jerarquías absolutas, sino con relaciones mucho más horizontales entre disciplinas, de tal manera que resulte posible aprovechar el conocimiento de otros campos y viceversa. En otras palabras, una organización colaborativa.

\section{El trabajo arquitectónico, la organización de la oficina}

Dos tipos de oficinas serán citadas, una «tradicional» y una «actual». Es importante decir que estas definiciones han sido establecidas sólo con el propósito de manifestar dos tipos de trabajo arquitectónico reconocibles ampliamente. El primer tipo es la manifestación de la oficina clásica, que ha dominado gran parte de nuestra historia, la del "gran maestro»; y la segunda como imagen de la oficina que hoy se está formulando en los círculos arquitectónicos más avanzados y especulativos, y que creemos manifiesta los senderos de desarrollo que nuestra disciplina seguirá enfrentando a futuro.

Durante siglos el paradigma del trabajo arquitectónico ha estado definido en torno al «maestro arquitecto» -cabeza creativa a cargo de tomar decisiones de diseños que luego son repartidas a sus subordinados-, como base de la creación de la obra arquitectónica. Este tipo de organización (árbol-raíz), ha tenido durante la historia como uno de sus más fundamentales aliados, la herramienta del dibujo bidimensional, básicamente porque esta técnica ha permitido siempre la (multi) copia rápida -sin grandes modificaciones- de la primera idea hacia los múltiples ayudantes. Durante la era industrial este esquema de trabajo alcanzó su punto culminante con la producción en masa, meta fundamental de la sociedad de ese entonces. La línea de producción Fordiana parecía reconocerse en casi todas las esferas. Claramente la oficina de arquitectura no estaba ajena a esta manera de entender los sistemas. Se conseguían los objetivos de manera bastante confortable y el maestro seguía a la cabeza de la organización, mientras que la transferencia de información desde éste lograba niveles de fluidez y velocidad inmejorables. Eficiencia y optimización emergían por defecto.

Hoy resulta frecuente escuchar en discusiones relativas al diseño, que diferentes tipos de colaboración son la manera en que la disciplina arquitectónica se desarrolla. Los cambios en la sociedad, junto con los crecientes avances tecnológicos, sin duda han cambiado la organización del conocimiento; pero también y completamente asociado a esto, han cambiado la configuración de la práctica. En palabras de Brett Steel: «Los territorios del diseño en redes de hoy en día, desafían las bases de lo que asumíamos debería ser el 'Arquitecto', criatura que existe cada vez menos como una figura localizable y estable -el individuo como diseñador intuitivo y 'creativo'- pero más como figura virtual, como organización en constante adaptación " ${ }^{6}$. Una «oficina actual» no debería necesitar -o al menos no debería depender exclusivamente-, de una cabeza en jefe a cargo de todas las ideas del proyecto, dado que puede -y hoy en día quizás debe-, ser alimentada desde diferentes campos de conocimiento de una manera completamente multidisciplinaria.

La aparición de diferentes tipos de colaboraciones basadas en equipos multidisciplinarios es la manifestación práctica del paradigma actual en arquitectura; múltiples experticias van alimentando al proyecto arquitectónico durante todo su proceso de desarrollo. El flujo de información en esta nueva oficina no es exclusivamente desde arriba-abajo (antiguo esquema árbol-raíz), sino que es mucho más horizontal entre todos los componentes de la red de colaboración. La naturaleza de las entradas y conexiones (relaciones) de la red de esta oficina de arquitectura se parecen más a la estructura rizomática de las raíces de algunos vegetales, o a las redes punto a punto (P2P) de Internet, más que a la rígida estructura de la metáfora árbol-raíz.

McLuhan, M. Understanding media: The extensión of man. Corte Madera, CA: Gingko Press, 2003.

3 Johnson, S. Bitmapping. Interface cultura: How new technology transform the way we create and communicate. New York, 1997; pp. 11-41.

"The crucial technological breakthrough lies in this idea of the computer as a symbolic system, a machine that traffics in representations or signs rather than in the mechanical cause-and-effect of the cotton gin or the automobile». / «El avance crucial reside en la idea del computador como un sistema simbólico, una máquina que opera con representaciones o símbolos, más que en el mecánico causa y efecto de una máquina desmontadora o un automóvil». (Traducción libre del autor). Johnson, S. Op cit., p. 15

Levi, P. Collective intelligence: Mankind's emerging world in cyberspace. (Traducción libre del autor). Cambridge, MA: Perseus Books, 1997; p. 10.

Steele, B. «Disappearance and distribution: The architect as machine interface». (Traducción libre del autor). Hunch: The Berlage Institute Report 2003; 6-7; pp. 422-36. 


\section{Nuevo mapa de relaciones, el rizoma}

Este cambio universal de paradigma en la organización del conocimiento adquiere una de sus definiciones más interesantes al explorar su estructura, instancia que nos permitirá enfrentarnos apropiadamente ante la búsqueda de comprensión de la organización disciplinar arquitectónica y su práctica, así como a las distintas relaciones existentes entre las partes participantes en los procesos y generaciones de diseño.

Uno de los documentos más importantes e influyentes en relación con la naturaleza de las nuevas organizaciones emergentes, en cuanto a conocimiento y sociedad, fue el trabajo desarrollado por Gilles Deleuze y Félix Guattari a finales del siglo pasado Mil mesetas: Capitalismo y esquizofrenia ${ }^{7}$ en donde aparece y se define la idea de rizoma.

El concepto de rizoma nace de la imagen (metáfora) de un tallo subterráneo absolutamente diferente en cuanto a organización, desarrollo y fisonomía, a la raíz y radículas comúnmente conocidas. «EI rizoma en sí mismo asume múltiples y diversas formas, desde ramificaciones en extensión en todas direcciones, hasta concreciones en bulbos y tubérculos "8. Estos rasgos físicos resultan fundamentales ya que definen las diferencias y contrastes entre la estructura del rizoma y la del árbol-raíz, pues ésta última "...infinitamente desarrolla la ley de que uno se hace dos, dos se hace cuatro y así... La lógica binaria es la realidad espiritual del árbol-raíz. Incluso disciplinas avanzadas como la lingüística mantienen el 'árbol-raíz' como imagen fundamental, y así se mantiene atada a las reflexiones clásicas. Esto apunta a decir que este sistema nunca ha alcanzado un entendimiento de la multiplicidad"9.

Para un mejor entendimiento del concepto, Deleuze y Guattari identifican 6 principios que definen la estructura de un rizoma:

1. Principio de conexión: «cualquier punto del rizoma puede estar conectado a cualquier otro, y debe estarlo. Esto es muy diferente al árbol o la raíz, ya que ellos trazan un punto, fijan un orden $»^{10}$.

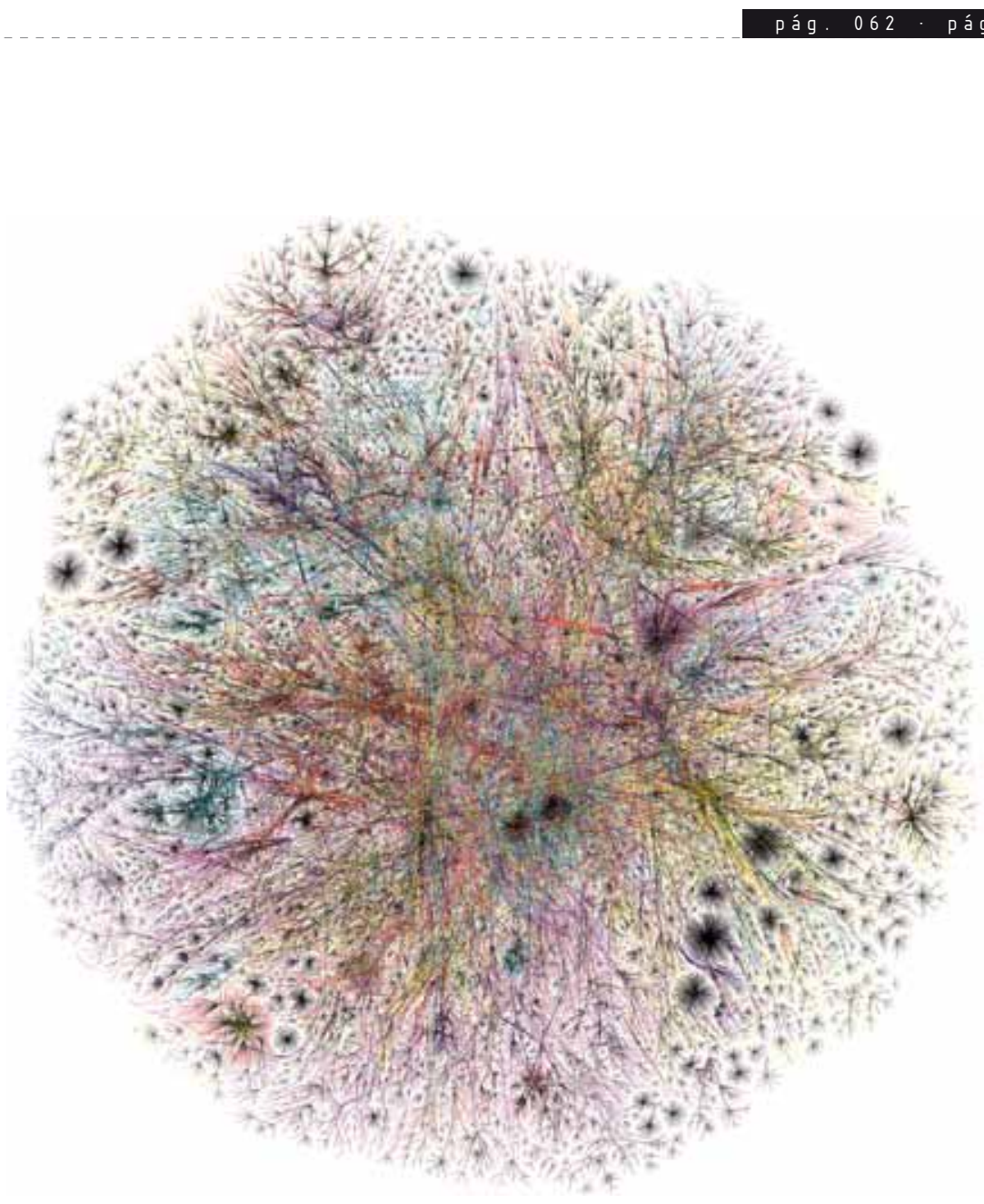

Mapa de las conexiones de Internet. Estructura rígida que permite conexiones rizomáticas [P.B. Mann (http://en.wikipedia.org/wiki//mage:Internet_map_4096.png)].

2. Principio de heterogeneidad: Dado que cualquier conexión es posible, el rizoma se despliega y desarrolla basado en la heterogeneidad; mientras que con el árbol-raíz (jerarquías en las relaciones), éstas resultan ser normadas por la homogeneidad (copia de la misma relación una y otra vez).

3. Principio de multiplicidad: El rizoma es siempre una multiplicidad que no puede ser reducida a una unidad, mientras que el árbol es un conjunto de elementos que puede ser reducido a ser un gran y único árbol. Con el rizoma «no hay unidad que sirva como pivote para una objetivación o subjetivación »"11, mientras que la estructura de árbol es basada en pivotes. "No hay puntos ni posiciones en un rizoma como los encontrados en la estructura del árbol o la raíz; sólo hay líneas» ${ }^{12}$. Así, dada su condición natural para establecer conexiones, el rizoma está siempre relacionado con el afuera; esto ya que «las multiplicidades se definen por el afuera: Por sus líneas abstractas, las líneas de fuga o desterritorialización, según las cuales ellas cambian su naturaleza y reconectan con otras multiplicidades ${ }^{13}$.

4. Principio de ruptura asignificante: El rizoma no supone ningún proceso

\footnotetext{
Deleuze, G., Guattari, F. «Rhizome». En: Thousand Plateaus: capitalism and schizophrenia. (Traducción Brian Massumi). London: Athlone Press, 1988; pp. 3-25.

Ibid., p. 7 (traducción libre del autor).

lbid . . 5 (traducción libre del autor).

lbid. p. 7 (traducción libre del at

11 lbid. p. 8 (traducción libre del autor).

12 Ibid., p. 8 (traducción libre del autor)

13 Ibid., p. 9 (traducción libre del autor)
} 
de significación ni jerarquías. Si bien es estratificado por líneas y por tanto «territorializado», organizado, significado, etc., estará siempre subyugado a las líneas de fuga que apuntan a nuevas e insospechadas direcciones. El rizoma es siempre un esquema, una cartografía que está siendo dibujada, en todo momento. «Un rizoma puede ser roto, destrozado en un punto dado, pero siempre empezará de nuevo desde una línea antigua, o desde una nueva» ${ }^{14}$.

5 y 6. Principio de cartografía y calcomanía: El rizoma puede ser mapeado, cartografiado, y esa cartografía mostraría siempre múltiples entradas; lo que significa que el rizoma puede ser abordado desde infinitos puntos, siendo así capaz, desde ahí, de referirse a cualquier otra zona del territorio. En otras palabras, «el árbol articula y jerarquiza calcos, copias. las copias son como las hojas de los árboles» ${ }^{15}$. Mientras que "el rizoma es del todo diferente, un mapa y no un calco. Lo que distingue al mapa del calco es que el primero está completamente orientado hacia la experimentación en contacto con lo real. El mapa no se reproduce inconscientemente, cerradamente hacia el mismo; el mapa construye el inconsciente. El mapa cultiva conexiones entre campos» 16

\section{La reorganización de la práctica arquitectónica}

Desde mediados de los noventa hemos estado presenciando claras manifestaciones de lo que podríamos denominar «práctica arquitectónica actual», en el más amplio sentido de la palabra; cubriendo desde la práctica profesional -por ejemplo, OCEAN, UNstudio, United Architects, Servo, etc.-, hasta el trabajo académico de varias escuelas de arquitectura, como la Architectural Association en Londres y su Master en Arquitectura «Design Research Lab». Todas estas organizaciones se presentan como claros referentes en el entendimiento del "principio de multiplicidad» y de «conexión», señalados bajo el prisma conceptual del rizoma, en cuanto aprovechan los distintos adelantos tecnológicos propios de la contemporaneidad en pro de organizar redes globales de práctica disciplinar; sumergiéndose en las distintas dinámicas del mundo actual (comunicaciones globales, economías de mercado globales, redes punto a punto, comunidades open source, web 2.0, etc.), y definiendo la práctica del diseño de manera impensable si la comparamos con la realidad de hace un par de décadas atrás. "Para la arquitectura estaría en juego la recalificación del diseño como un acto de negociación, simultáneamente más intrínseca y más extrínseca a la tradicional noción de

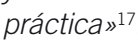

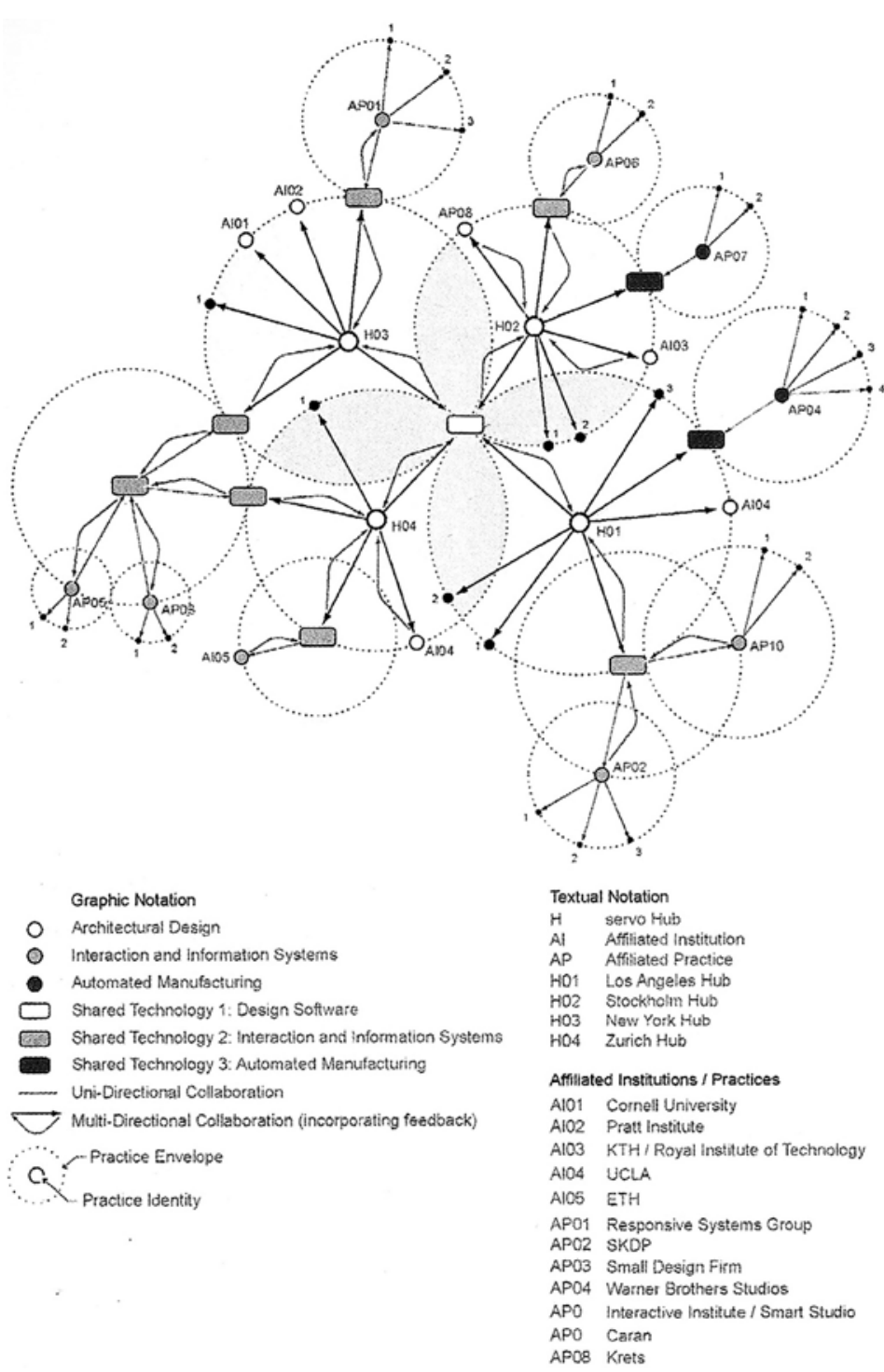

Diagrama de organización de la red de trabajo colaborativo de Servo. [Aaron White (Erdman, D. «Parallel processing: Design practice». Architectural Design 2006; 76(5): p. 86 )] 
Estas nuevas organizaciones y maneras de organización que se han ido adoptando, no se basan sólo en un cambio en las relaciones entre las personas envueltas en el desarrollo de un proyecto arquitectónico; sino que implican más bien la adopción de recursos e infraestructura de telecomunicaciones, así como de los últimos adelantos en materia de software y dispositivos electrónico/ computacionales, los que soportan estas nuevas estructuras relacionales y así la generación de este cambio en la práctica de arquitectura.

El potencial de la tecnología de telecomunicaciones, en términos de hacer posible el espacio virtual abierto para la reunión de múltiples agentes, sin importar dónde estén ubicados en el mundo, resulta un factor clave para entender lo que realmente significa -y puede llegar a significar- una red hoy en día. Las redes hipervinculadas (hyperlinked networks) hacen posible entender la expresión multiplicidad de manera más amplia que nunca, comprendiendo la práctica de diseño colaborativo como sólo una de las múltiples (y cada día cambiantes y crecientes) organizaciones nacidas durante los últimos años.

«Estamos presenciando una combinación de redes de comunicaciones y redes sociales. Cada vez que una nueva tecnología de comunicaciones baja el umbral para el actuar colectivo de los grupos, nuevos tipos de instituciones emergen »18.

«Softwares sociales, que soportan comunicaciones grupales (desde listas de e-mail a blogs, Wikis o los masivos juegos en línea de multijugadores) tienen una dinámica que no puede ser entendida desde el agente individual. Sus operaciones son colaborativas, sensibles y emergentes» ${ }^{19}$

De tal manera, y comprendiendo su potencial colectivo, o lo que también es llamado como Cognición Colectiva ${ }^{20}$, podemos extrapolar estos potenciales de la red principal con que hoy interactuamos -Internet-, al del trabajo de la oficina de arquitectura.

Un interesante ejemplo de cognición colectiva basado en las redes hoy existentes, es el caso del proyecto de 1998 «Aegis hyposurface»,
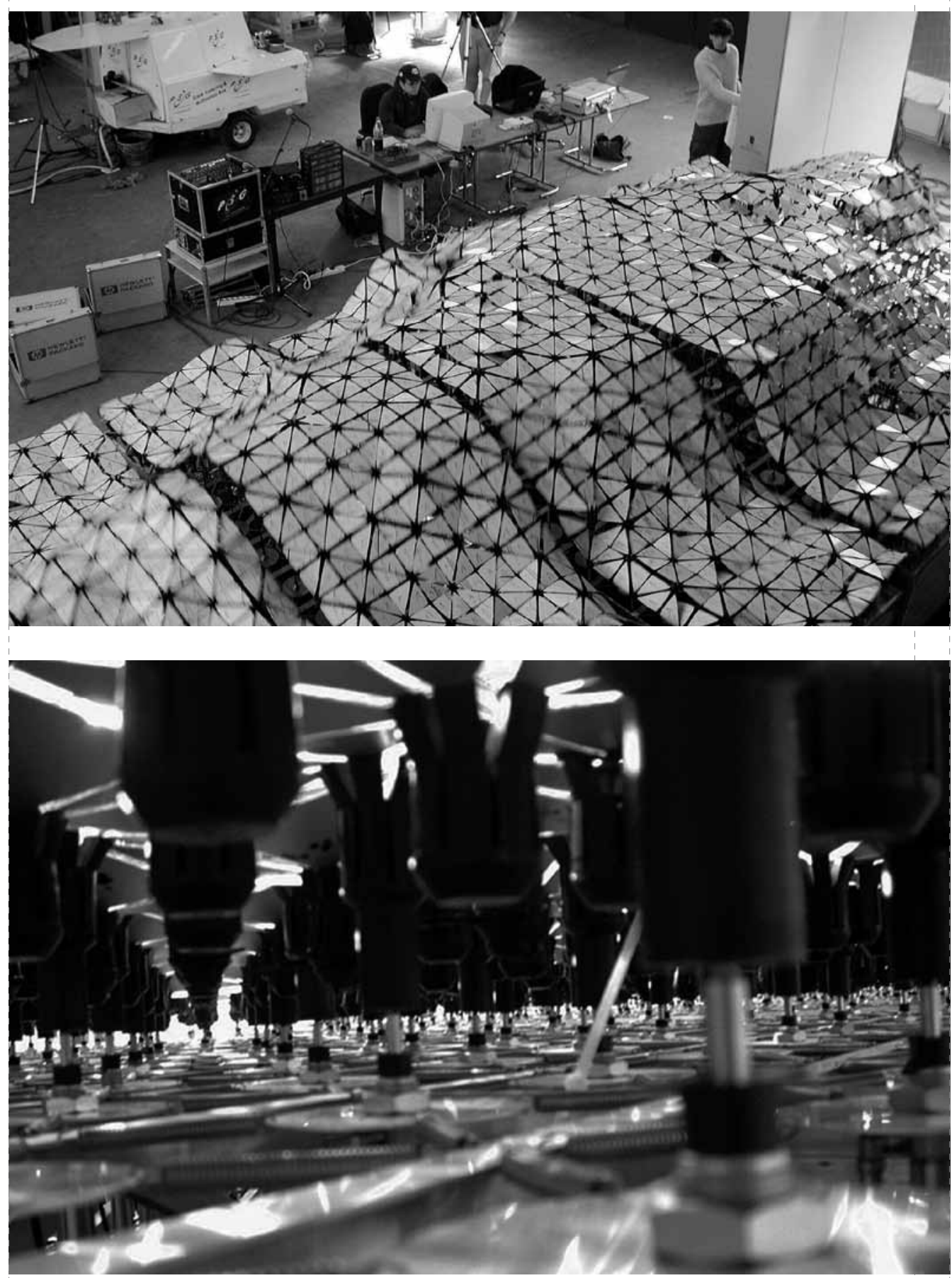

Proyecto «Aegis hyposurface». [ARS ELECTRONICA archive (http://www. aec.at/de/archives/ picture_ausgabe_02_new.asp?iArealD=6\&showArealD=76)]

18 Erdman, D., et. al. «Parallel processing: Design/Practice». (Traducción libre del autor). Architectural Design 2006; 76(5): p. 84.

19 Tierney, T. «Collective cognition: Neural fabrics and social software». (Traducción libre del autor). Architectural Design 2006; 76(5): p. 39.

20 Ibid.

«Cognición colectiva» es definida como: «Una serie de agentes participando de un cuerpo de conocimiento, y por extensión, beneficiándose de la información del grupo».

(Traducción libre del autor). 
diseñado por la firma dECOI. En este proyecto la colaboración multidisciplinaria y la idea de red fueron llevadas al extremo. Participaron arquitectos, matemáticos, programadores, ingenieros estructurales, ingenieros eléctricos, profesionales en balística, profesionales en neumática y más; distribuidos prácticamente alrededor de todo el mundo. En ese momento, "el 'e-mail' fue el medio de comunicación por defecto, y en la ausencia de más formales sistemas de manejo y captura de conocimiento, el principal y más común recurso del proyecto ${ }^{21}$. Hoy en día, si bien el e-mail sigue siendo un medio fundamental de comunicación, las organizaciones colaborativas se organizan principalmente con dos tipos de redes en línea, la punto a punto y la cliente/ servidor $^{22}$. Basado en este tipo de redes, un interesante ejemplo de organización colaborativa en el campo del diseño es el caso del Spatial Information Architectural Laboratory (SIAL ${ }^{23}$ ). El centro está ubicado en Melbourne, Australia, en la Universidad RMIT; es dirigido por Mark Burry y está a cargo del proyecto de continuación de la iglesia Sagrada Familia, de Antoni Gaudi, en Barcelona. Desde el año 2002 esta organización grupal está trabajando con un Wik $R^{4}$ como plataforma que usan para varias áreas del trabajo (desde trabajo académico de pre y posgrado hasta actividades profesionales), pero en el proyecto de la Sagrada Familia resulta asombroso el aumento del potencial de esta herramienta como plataforma de diseño colaborativo. Andrew Burrow y Jane Burry explican, "nosotros trabajamos juntos en proyectos como la Sagrada Familia donde el equipo está distribuido internacionalmente. Por cada dos de cada seis u ocho semanas, Mark Burry trabaja en terreno en Barcelona, mientras otros miembros del SIAL continúan trabajando en el proyecto en Australia. El Wiki es el foro fundamental para compartir reuniones, tomar decisiones mientras eventos ocurren en la obra, y para discutir respuestas o modelos preparados en Melbourne. La página del Wiki puede tan fácilmente ser organizada en torno a proyectos particulares del proyecto mayor, o en torno a visitas específicas, temas y colaboradores ${ }^{25}$

Diagrama de conexiones del Wiki usado por SIAL. Cada nodo es una página, cada línea es un vínculo entre páginas. [Burrow, A. «Working with wiki, by design». Architectural Design 2006; 76(5): p. 96)]

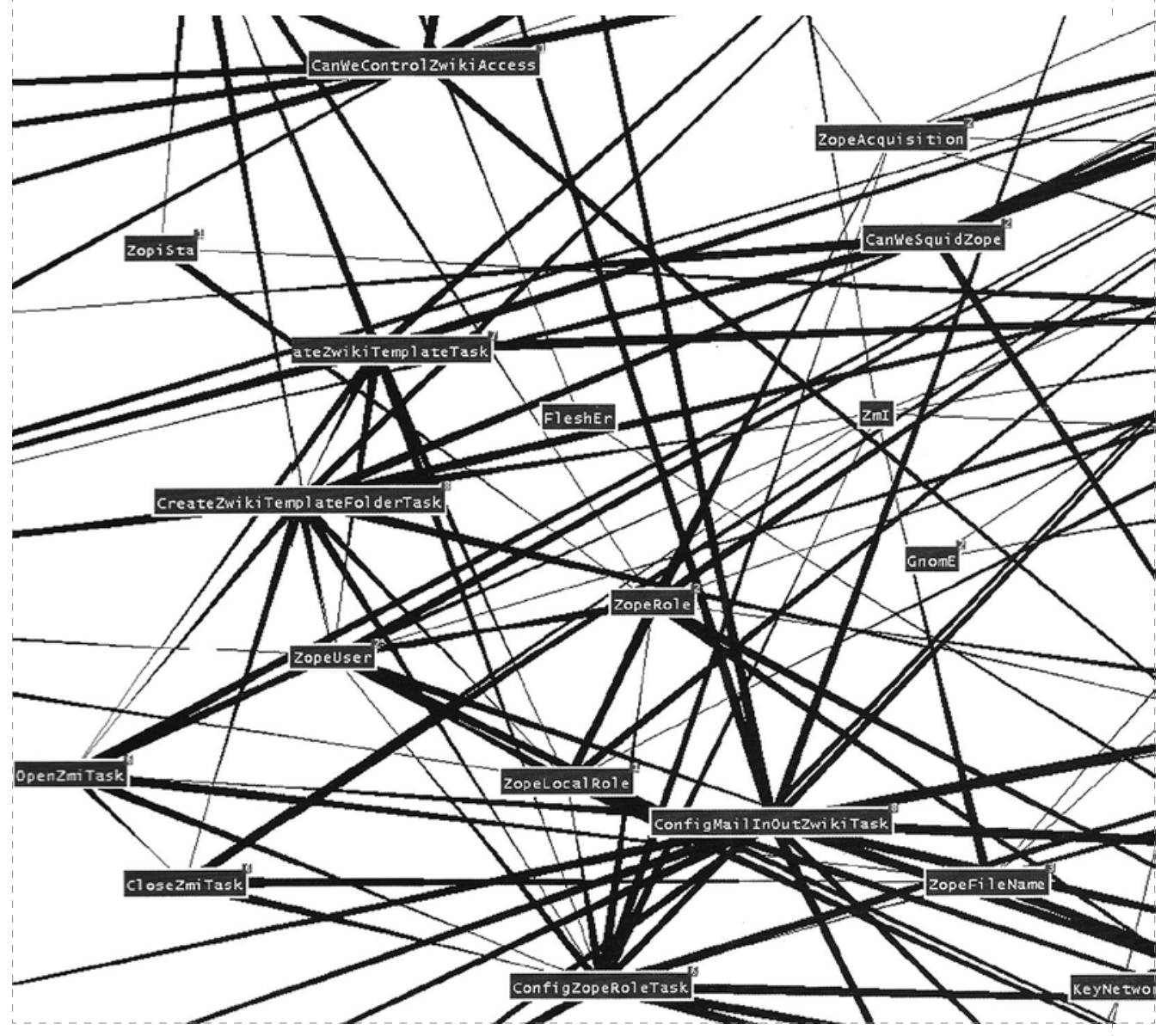

Este último ejemplo manifiesta que la «oficina actual» no necesita un lugar fijo en el mundo para operar de manera adecuada de hecho es capaz de desempeñarse con extrema eficiencia y calidad (de manera muy global, pero también por defecto lo contrario, muy local), sin puntos fijos en la red. Esta oficina necesita ser capaz de cambiar a cada momento si es necesario («ruptura asignificante») y necesita ser capaz también de reorganizarse cuando sea necesario, durante un proyecto específico o durante la vida de la misma -múltiples proyectos(«cartografía y calcomanía»). Pero el ejemplo del Wiki es sólo uno de tantas posibilidades. Hoy en día los avances tecnológicos permiten a la oficina de arquitectura manejar su flujo de información mediante innumerables dispositivos electrónicos inalámbricos (desde computadores portátiles, pasando por toda la gama de PDA, hasta los teléfonos móviles actuales), materializando así redes flexibles, pero a la vez suficientemente poderosas como para soportar infinitud de entradas (inputs) ubicados alrededor del mundo y en línea. De esta manera, creando estructuras colaborativas hiperconectadas de flujo de conocimiento, de una manera nunca antes vista; traspasando los límites de múltiples disciplinas y aumentando así la producción arquitectónica, pero quizás más importante aún, aumentando las posibilidades creativas de la disciplina. «Las

Burrow, A., Burry, J. «Working with Wiki, by design». (Traducción libre del autor). Architectural Design 2006; 76(5): p. 97.

Topológicamente diferentes, sus principales características son que en la primera red cada agente puede operar como cliente o servidor, y en la segunda siempre se tiene un servidor que sirve a todos los agentes/clientes, además que la segunda puede operar a mayor escala y con mayor seguridad. http://www.sial.rmit.edu.au/

Burrow, A., Burry, J. Op cit, p. 98.

«Wiki es una serie de páginas web vinculadas que generalmente están abiertas a todo quien quiera contribuir y editarla. Es un 'entramado' más que una estructura de 'árbol', lo que significa que soporta mayoritariamente vínculos laterales. A través de los nombres de las páginas y los vínculos de hipertexto manifiesta tácitamente su estructura. Crea y provee acceso a la historia del proyecto y simultáneamente genera un mapa de los intereses de la comunidad». (Traducción libre del autor)

Ibid. (Traducción libre del autor). 
redes cambian todo el tiempo. De hecho, 10 que les da a las redes su perfil productivo es el aprendizaje mutuo, el intercambio de conocimiento y habilidades, y particularmente en el trabajo del diseño, que funciona desde las interacciones y colaboraciones cambiantes. La colaboración mediante redes está por lo tanto coevolucionando hacia el desarrollo individual y colectivo, facilitando así la producción de un diseño original»²6.

Como explicamos, la naturaleza de las redes (estructura y tecnología) y las posibilidades que dan (compartir archivos digitales, comunicación en línea, movilidad, colaboración, etc.), son fundamentales en la «oficina actual» de arquitectura. Sin embargo, hay al menos un cambio más necesario para alcanzar un estado real de redes fluidas y colaborativas en el diseño arquitectónico: Las plataformas de diseño. Desde este punto de vista no es nuevo decir que los softwares CAD de modelado y alzado digital son fundamentales para la arquitectura hoy, pero en cuanto a las redes su importancia no radica en sus características intrínsecas más conocidas (control de geometrías complejas, capas, renders, animaciones, almacenado de datos, etc.), sino la capacidad del archivo digital (y del modelo 3D) de soportar cambios a través de un proceso arquitectónico (el proceso de diseño mismo, más las múltiples etapas de fabricación y construcción), y desde múltiples direcciones (inputs). Si la "oficina tradicional» basaba su trabajo de diseño en la plataforma del dibujo bidimensional y la multicopia de él («calco»), la «oficina actual» lo hace basado en el archivo digital y el modelo 3D; ya que el demandante flujo de información, así como las constantes modificaciones del proyecto, necesitan un soporte flexible durante estos diferentes procesos y etapas.

El modelo digital 3D puede soportar las modificaciones y entradas de múltiples agentes en una red colaborativa con eficiencia y en tiempo real, ya que tiene las propiedades de un mapa («ruptura asignificante», «cartografía y calcomanía») porque está compuesto por vectores y parámetros, principal diferencia con el dibujo bidimensional de proyecciones, el que opera como una imagen raster, una serie de líneas y puntos perpendiculares fijos capaces de ser copiados, pero no modificados más allá de sus características propias (posición, tamaño relativo, número, etc.), el que necesariamente resulta incapaz de soportar la cantidad de información de un proyecto actual. En este contexto, con el computador el proyecto se basa en modelos digitales 3D, donde los objetos son colecciones de comandos, opuesto a lo que sucede con los dibujos bidimensionales, en que el proyecto es la resultante de una serie de proyecciones aisladas. Esto significa que el modelo digital 3D funciona por acumulación; todo está guardado, los elementos arquitectónicos están compuestos por información, la que para el caso de las oficinas y softwares más avanzados, son datos y parámetros completamente relacionados (constricciones) entre ellos -softwares paramétricos-, borrando así por completo las tradicionales jerarquías de diseño basadas en la lectura unidirecciona desde lo general al detalle. Como dice Stan Allen, "si el poder del computador radica en su capacidad de manejar grandes cantidades de información, múltiples variables, y códigos abstractos, es importante estar atentos a la emergente sensibilidad por paradigmas de organizaciones más flexibles y diagramáticos: una abstracción 'condicional' y contingente. Esto en cambio implica un cambio desde las falsas certezas de la visualización hacia las capacidades generativas del computador como máquina abstracta» ${ }^{27}$; máquina que por su naturaleza es capaz de plantearse como una plataforma fundamental para la originalidad en el diseño, pero necesariamente cambiando la manera de concebirlo.

En los software CAD, el dibujo bidimensional de proyección resulta ser uno más de los productos que uno puede extraer del modelo 3D. Básicamente el modelo 3D opera como elemento de diseño, y los planos como elemento de representación del momento en que se extraen. Esto puede significar para la «oficina actual» que al trabajo en red se le puede sumar este modelo 3D, el que funcionaría como "archivo servidor», en el que todos los componentes de esta red podrían trabajar desde diferentes locaciones y tiempos. Así, el modelo opera como manifestación cambiante del estado de «negociación» de los diferentes participantes de la red. Una situación muy representativa de esta manera de operar es el relativamente reciente cambio de los softwares CAD a softwares paramétricos por un lado, con la inclusión de la disciplina de la programación en el diseño, y por otro por la aparición de los softwares BIM ${ }^{28}$, los que permiten a los arquitectos almacenar toda la información de un proyecto en un solo archivo con todas las propiedades ya descritas (diseño, fabricación, construcción, especialidades, etc.). Quizás en este ámbito uno de los arquitectos que ayudó a dar el paso fundamental fue Frank Gehry y sus proyectos de fabricación y construcción compleja; y no sólo por su posterior asociación con una compañía de softwares BIM (CATIA), sino por los avances en el rol del modelo 3D a través de todo el proceso de diseño, desde el inicio hasta la parte culminante de la fabricación y construcción de los edificios. En este caso el modelo 3D utilizado opera como 'modelo maestro', y es la plataforma para todas las decisiones de diseño. "El modelo maestro de CATIA es la única fuente de información para el diseño del edificio, y se transforma en parte legal de los documentos del contrato. Si bien puede tomar cualquier forma, su condición base es una modelo wireframe, base de datos tridimensional desde la cuál se extrae toda la información dimensional del proyecto ${ }^{29}$.

Por último, las exigencias tanto de innovación como de originalidad características del mundo contemporáneo, están empujando mucho más allá los límites ya borrosos de la disciplina y práctica arquitectónica, y depende de la capacidad del equipo interdisciplinario que lleve a cabo un determinado proyecto lograr un uso provechoso de estos cambios de paradigma, y desarrollar su potencial incluso hacia puntos más lejanos. Quisiéramos finalmente reconocer que ciertamente la anteriormente llamada «oficina actual» no es de aplicación generalizada aún, pero es incluso más patente y cierto que estos tremendos procesos de adaptación y cambio disciplinar están ocurriendo precisamente en el día de hoy.

26 Hensel, M. «Evolving synergy: OCEAN currents, current OCEANs and why networks must displace themselves». (Traducción libre del autor). Architectural Design 2006; 76(5): p. 108.

27 Allen, S. «Terminal velocities: The computer in the design studio». En: The virtual dimension. (Traducción libre del autor). Nueva York: Princeton Architectural Press, 1998; p. 248.

28 El Building Information Model (BIM) es un modelo digital que almacena toda la información sobre un edificio. Integra la información geométrica, funcional, especialidades, etc., en un solo sistema interrelacionado.

29 Lindsey, B. Digital Gehry. Material Resistance/Digital Construction. (Traducción libre del autor). Basel: Birkhauser, 2001; p. 87. 

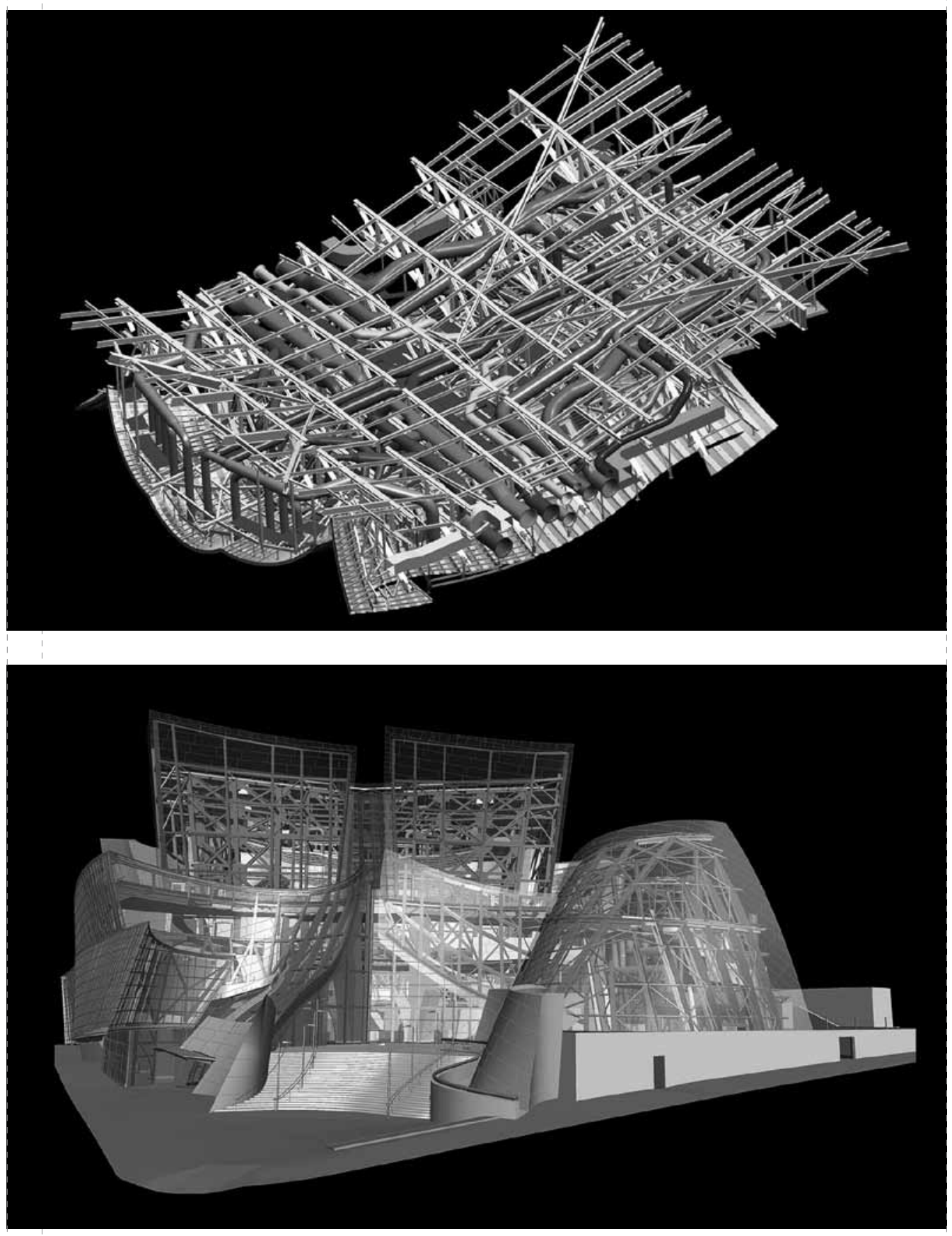

Modelo 3D BIM Software Gehry Technologies. [Gehry Technologies / Gehry Partners, LLP (http: //gehrytechnologies.com/company-press-03-23-2006.html)] .

\section{Bibliografía}

Libros

Allen, S. «Terminal velocities: The computer in the design studio». En: The virtual dimension. Nueva York: Princeton Architectural Press, 1998; pp. 242-255.

Deleuze, G., Guattari, F. Thousand Plateaus: Capitalism and schizophrenia. (Traducción Brian Massumi). Londres: Athlone Press, 1988.

Johnson, S. Interface cultural: How new technology transform the way we create and communicate. Nueva York, 1997.

Levi, P. Collective intelligence: Mankind's emerging world in cyberspace. Cambridge, MA: Perseus Books, 1997; p. 10.

Lindsey, B. Digital Gehry. Material Resistance/ Digital Construction. Basel: Birkhauser, 2001

McLuhan, M. Understanding media: The extensión of man. Corte Madera, CA: Gingko Press, 2003.

Velázquez, P. «Experimentación transversal en arquitectura: un reconocimiento a los nuevos paradigmas de la posmodernidad». Seminario de Investigación FAU. Santiago: Universidad de Chile, 2004.

Zellner, P. «OCEAN, Global». En: Zeller, P. Hybrid space. New forms in digital architecture. New York: Rizzoli International Publications Inc, 1999; pp. 150-163.

\section{Revistas}

Architectural Design 2006; 76(5)

Hunch: The Berlage Institute Report 2003; 6 y 7.

Hunch: The Berlage Institute Report 2005; 9.

VERB: Architectural Boogazine 2001; 1.

Sitios web

http://www.sial.rmit.edu.au/

http://www.sial.rmit.edu.au/Projects/Aegis Hyposurface.php

http://www.pcmech.com/show/network/126/ http://en.wikipedia.org/

http://www.gsd.harvard.edu/research/publications/ hdm/back/21_aravena.html 